\title{
Field-Strength Depression of Emitters Dispersed from a Point Source into a Cylindrical Volume
}

\author{
Palmer G. Steward \\ Retired Medical Physicist 5234 Nobis Ct., Davenport, Iowa, USA \\ Email: psteward@earthlink.net
}

How to cite this paper: Steward, P.G. (2021) Field-Strength Depression of Emitters Dispersed from a Point Source into a Cylindrical Volume. Journal of Applied Mathematics and Physics, 9, 545-555. https://doi.org/10.4236/jamp.2021.94038

Received: January 29, 2021

Accepted: March 30, 2021

Published: April 2, 2021

Copyright $\odot 2021$ by author(s) and Scientific Research Publishing Inc. This work is licensed under the Creative Commons Attribution International License (CC BY 4.0).

http://creativecommons.org/licenses/by/4.0/

\section{(c) (i) Open Access}

\begin{abstract}
Dispersing emitters, whose emissions are consistent with the inverse square law, from a point into cylindrical shapes causes a field-strength depression which is strongly dependent upon the cylinder's dimensions and the distance from the cylinder. The computations of field-strength depressions are partitioned into the components due to dispersion of the emitters alone, due to self-absorption alone, and the total field-strength depression. Each partition is displayed graphically for distances of $5 \mathrm{~cm}$ to 10 meters from the cylinders and for a variety of cylinder dimensions. The shape of the displayed curves is analyzed to reveal the basis for correlations between curve shape and the cylinder dimensions. Practical use of the data presented graphically is demonstrated.
\end{abstract}

\section{Keywords}

Cylindrical Shapes, Radionuclide Emissions, Self-Absorption Component, Dispersion Component, Inverse Square Law

\section{Introduction}

Normally one feels compelled to focus one's research such that it will be viewed as beneficial in the context of current concerns. A common task for the medical radiation physicist is to estimate the radiation field strength in the vicinity of a patient in whom radiation emitters are dispersed. The motivation for initiating this project was to deal with this issue. First, guidance for managing the radiation safety for families of patients undergoing ${ }^{131}$ I therapy was developed [1]. Due to the ease with which the point-source calculation is performed there has been temptation to use it, even though it is a poor approximation, at least in the 
case of a bystander being in close proximity to the radionuclide patient under treatment. To deal with this issue, point source correction factors for conveniently determining the field strength in close proximity to cylinders (representing patients) in which the emitter distribution may not be uniform were presented [2]. This later paper dealt only with emitters distributed in the body of the patient, not with emitters localized in the patient's thyroid. Possibly the most comprehensive achievement regarding this issue is a paper directed specifically toward medicine and public health [3]. In this paper complex biokinetic modeling tracks the temporal flow of ${ }^{131} \mathrm{I}$ emitters in patients with three different biokinetic behaviors. Using the Monte Carlo method in conjunction with a patient model containing realistically placed glands, organs and with movable arms and legs, it then computes resultant effective doses to bystanders, similarly modeled, at separation distances $10 \mathrm{~cm}$ to 3 meters. The limitations of the point-source approximation are demonstrated by comparing these results to those using only a point-source calculation.

This current paper is a departure from those whose primary focus is directed toward dealing with a matter of current practical concern. Rather the primary focus is on interesting phenomena revealed while using mathematical processes developed for previous works [1] [2] to analyze the depression of the field strength by dispersing emitters confined to a point source into uniform distributions throughout cylinders of 10 to $40 \mathrm{~cm}$ diameters and 10 to $200 \mathrm{~cm}$ lengths. The field-strength depressions are presented at distances of $5 \mathrm{~cm}$ to $10 \mathrm{~m}$ from the cylinders. The component of field-strength depression due only to source dispersion is presented separately from the depression component due to self-absorption within the cylindrical volume. The depression due only to dispersion is valid for all emitters consistent with inverse-square behavior, while self-absorption is characterized by using a 10-cm HVL for emissions within the cylinder's absorbing medium. The total depression is presented as the product of these two components.

By presenting field strengths as a depression of the field strength from that of a point source something of practical value becomes available-the actual field strength from the cylindrical volume is simply the product of the field-strength depression presented below and a simple point source computation for a point on the proximal surface of the cylinder.

\section{Methods}

The method used here for computing the field strength of emitters confined to a cylindrical volume was previously described in detail [2]. Briefly the cylinder is filled with line sources. Line sources are parallel with the cylinder's axis of symmetry, have the length of the cylinder, and are spaced throughout the cylinder at 5 -mm intervals. Specifically, one line source is placed at the axis of symmetry and other sources are placed at 5-mm intervals along concentric circles with radii of adjacent circles differing by $5 \mathrm{~mm}$. As a result the $10-\mathrm{cm}$ diameter cylinder 
contains 337 line sources, the $25-\mathrm{cm}$ cylinder has 2,017 line sources, and the $40-\mathrm{cm}$ cylinder has 5111 line sources. The placement of line sources within a $25-\mathrm{cm}$ diameter cylinder is illustrated in Figure 1, which depicts the right-hand half of the cylinder's cross-section. This project, at the outset, was constrained by the assumption that there was right to left symmetry of the cylinder. This permits the total field strength to be computed by doubling the contribution of each right hand line source and adding that of the single central source. The location of the point source from which the emitters are dispersed, causing the field-strength depression, is indicated in Figure 1. Distributing the emitters throughout the cylinder in this manner has assisted in demonstrating the effects of certain nonuniformities in emitter distributions, and it assists the nonmathematical reader to conceptualize the numerical integration process.

Using this configuration of the cylindrical volume the field strength (air kerma) has been shown to be [2],

$$
K_{a}(r)=\frac{24 \Gamma T_{1 / 2 \mathrm{eff}}}{\ln (2)} \sum_{i=1}^{n(r)} N_{i} \int_{-L / 2}^{L / 2} P_{i}(l) \frac{\mathrm{e}^{-\left[\frac{\ln (2) \Delta g_{i} \sqrt{l^{2}+g_{i}^{2}}}{H V L} g_{i}\right.}}{l^{2}+g_{i}^{2}} \mathrm{~d} l
$$

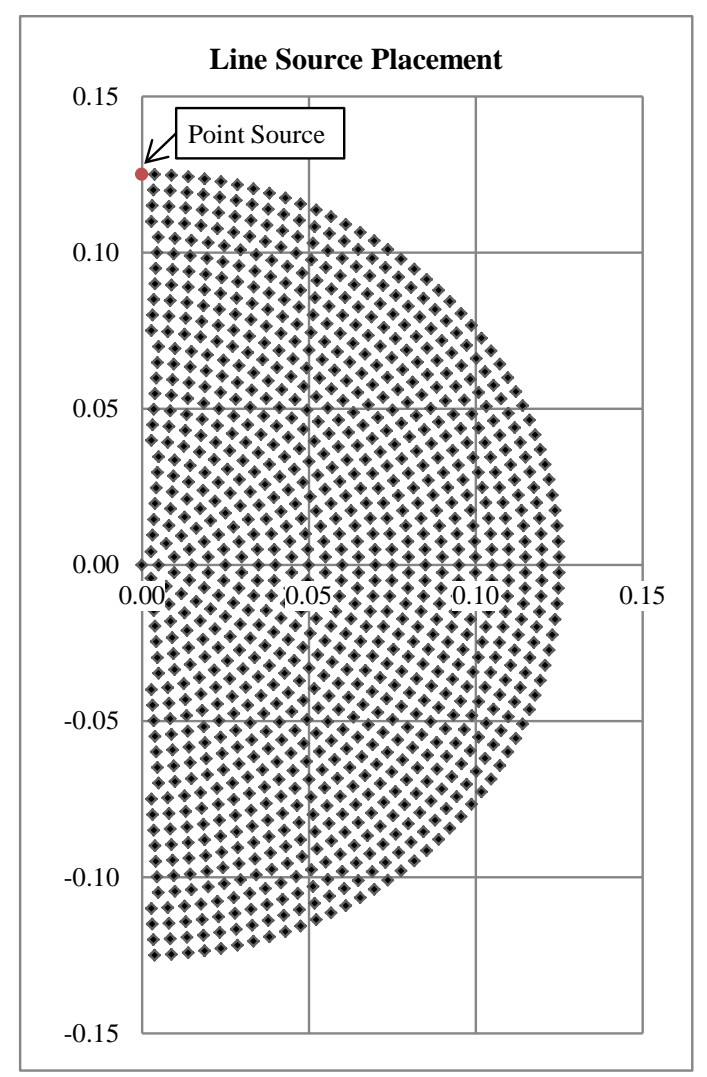

Figure 1. Depicts the right hand half of a $25-\mathrm{cm}$ cylinder's midplane cross section (perpendicular to its axis of symmetry). Placement of the line sources in a Cartesian coordinate system whose origin is at the cylinder's center is shown. Because there is left-right symmetry, only the right hand half appears. Distances along the axes are in units of meters. The position of the point source whose activity to which the field-strength depressions are referenced is shown in red. 
where $\Gamma$ is the air kerma rate constant for the emitting nuclide, $T_{1 / 2 \text { eff }}$ is the emitter's effective half-life including both biochemical processes and nuclear decay, $n(r)$ is the total number of line sources in a cylinder of radius $r, N_{i}$ is the emitter's activity for the $i^{\text {th }}$ line source, $L$ is the length of the $i^{\text {th }}$ line source (i.e., the cylinder's length), $P_{i}(l)$ is the activity per unit length at position $l$ along the $i^{t h}$ line source normalized such that $\int_{-L / 2}^{L / 2} P_{i}(l) \mathrm{d} l=1, \Delta g_{i}$ is the distance perpendicular to the $i^{\text {th }}$ line source from source to emission-exit from the cylinder, $H V L$ is the half value length for emissions in the absorbing medium of the cylinder, $l$ is the emission's position along the $I^{\text {th }}$ line source, $g_{i}$ is the distance perpendicular to the $i^{\text {th }}$ line source from source to the field-strength assessment point on the $y$ axis of Figure 1. Note that if $\left(x_{i}, y_{i}\right)$ are coordinates of the $i^{\text {th }}$ line source in the Cartesian coordinate system of Figure 1, $g_{i}$ is related to the size of the cylinder and the distance to the field-strength assessment point by

$$
g_{i}=\sqrt{\left(d+r-y_{i}\right)^{2}+x_{i}^{2}},
$$

where $r$ is the cylinder's radius, and $d$ is the distance from the proximal surface of the cylinder ("Point Source" of Figure 1) to the field-strength assessment point on the $y$ axis.

An unshielded point source centered on the proximal surface of the cylinder (indicated in Figure 1) delivers a field strength

$$
K_{a}=\frac{24 \Gamma N_{0} T_{1 / 2 \mathrm{eff}}}{d^{2} \ln 2}
$$

where $N_{0}=\sum_{i=1}^{n(r)} N_{i}$. Dividing Equation (1) by Equation (3), the field-strength depression $^{1}$ due to dispersing the emitters from the point source into the cylinder is

$$
D_{p \rightarrow c}=\frac{d^{2}}{N_{0}} \sum_{i=1}^{n(r)} N_{i} \int_{-L / 2}^{L / 2} P_{i}(l) \frac{\mathrm{e}^{-\left[\frac{\ln (2) \Delta g_{i}}{H V L} \sqrt{l_{i}^{2}+g_{i}^{2}}\right]}}{l^{2}+g_{i}^{2}} \mathrm{~d} l .
$$

Since the emitters are distributed uniformly throughout the cylinder, the activity along each line source is constant, $P_{i}(l)=1 / L$, and the activity of each line source is $N_{i}=N_{0} / n(r)$. The total field-strength depression then simplifies to

$$
D_{p \rightarrow c}=\frac{d^{2}}{n(r) L} \sum_{i=1}^{n(r)} \int_{-L / 2}^{L / 2} \frac{\mathrm{e}^{-\left[\frac{\ln (2) \Delta g_{i}}{H V L} g_{i} \sqrt{1^{2}+g_{i}^{2}}\right]}}{l^{2}+g_{i}^{2}} \mathrm{~d} l
$$

The integrand of Equation (5) is integrated numerically. However, if there is no self-absorption ( HVL $\rightarrow \infty$ ) the numerator of the integrand becomes 1.0, the expression is then integrable in closed form and Equation (5) evaluates to

${ }^{1}$ Dividing the field strength of a cylindrical source (Equation (1)) by the field strength of a point source with the same emitters (Equation (3)), results in an expression for the cylindrical source strength relative to that of the point source. This quotient is always less than 1.0, and it is referenced in this paper as a depression of the point-source strength. To avoid confusion, note: as the depression increases, the relative source strength decreases and vice versa. 


$$
D_{p \rightarrow c}^{\text {no abs }}=\frac{2 d^{2}}{n(r) L} \sum_{i=1}^{n(r)} \frac{\tan ^{-1} \frac{L / 2}{g_{i}}}{g_{i}},
$$

where $g_{i}$ is given by Equation (2). Equation (6) is the component of the field-strength depression due only to the geometric dispersion of point source emitters uniformly throughout the cylinder. The field-strength depression due to self-absorption alone is Equation (5) divided by Equation (6):

$$
D_{p \rightarrow c}^{a b s \text { alone }}=\left[2 \sum_{i=1}^{n(r)} \frac{\tan ^{-1} \frac{L / 2}{g_{i}}}{g_{i}}\right]^{-1} \sum_{i=1}^{n(r)} \int_{-L / 2}^{L / 2} \frac{\left.\mathrm{e}^{-\left[\frac{\ln (2) \Delta g_{i}}{H V L} \sqrt{l^{2}+g_{i}^{2}}\right.}\right]}{l^{2}+g_{i}^{2}} \mathrm{~d} l .
$$

\section{Results and Discussion}

Dispersing isotropic emitters from a point source into cylindrical shapes causes a reduction in the field strength. This field-strength depression is strongly dependent upon the cylinder's dimensions and the distance at which the field strengths are assessed. Figures 2-10 show the magnitude of this depression over distances from $5 \mathrm{~cm}$ to 10 meters and for cylinders of varying lengths and diameters. Field-strength depressions are shown separately for that component of the depression due to only dispersal of the emitters (Equation (6)), and for the depression due to only self-absorption of the emissions in the absorbing medium of the cylinder (Equation (7)). The total field-strength depression (the product of these two) is also shown (Equation (5)). Self-absorption is computed using a $10-\mathrm{cm} \mathrm{HVL}$. (This is the broad-beam HVL in water for the $365-\mathrm{keV}$ photon from ${ }^{131}$ I decay [4].) Because the HVL for a broad beam is used, when cylinder dimensions approach $10 \mathrm{~cm}$ the depression may be a slight overestimate for ${ }^{131} \mathrm{I}$ decay, but general trends of field-strength depression regarding cylinder dimensions and distances still will be valid.

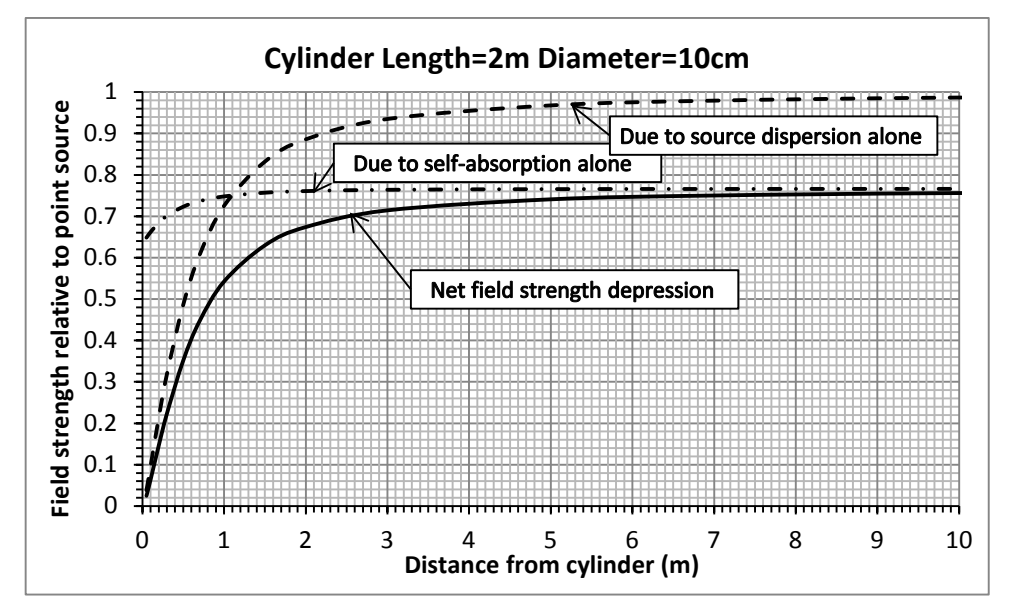

Figure 2. The field-strength depression due to dispersing emitters from a point source into a $10-\mathrm{cm}$ diameter cylinder 2 meters long. Emissions are assumed consistent with the inverse-square law. Absorption is computed using $\mathrm{HVL}=10 \mathrm{~cm}$. 


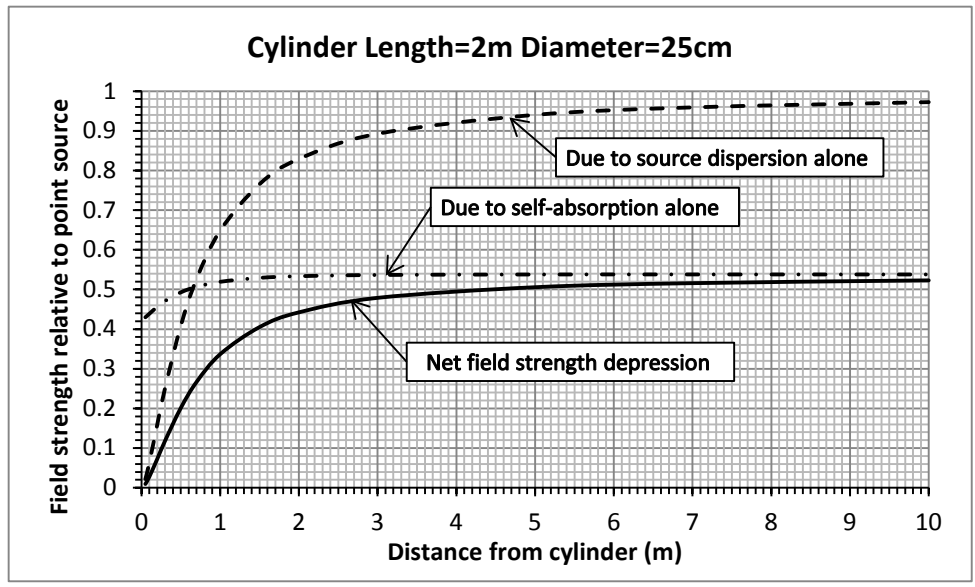

Figure 3. The field-strength depression due to dispersing emitters from a point source into a $25-\mathrm{cm}$ diameter cylinder 2 meters long. Emissions are assumed consistent with the inverse-square law. Absorption is computed using $\mathrm{HVL}=10 \mathrm{~cm}$.

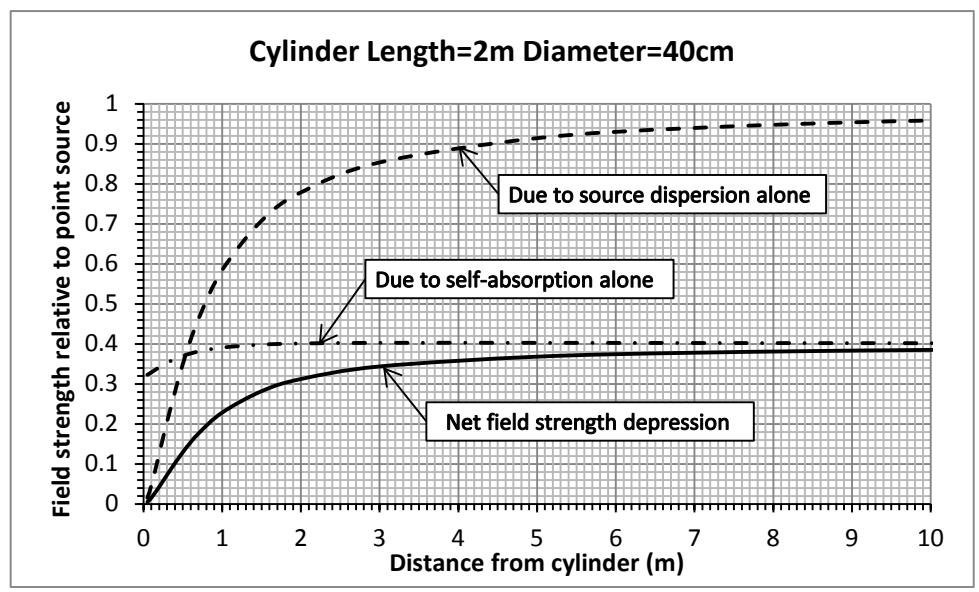

Figure 4. The field-strength depression due to dispersing emitters from a point source into a $40-\mathrm{cm}$ diameter cylinder 2 meters long. Emissions are assumed consistent with the inverse-square law. Absorption is computed using $\mathrm{HVL}=10 \mathrm{~cm}$.

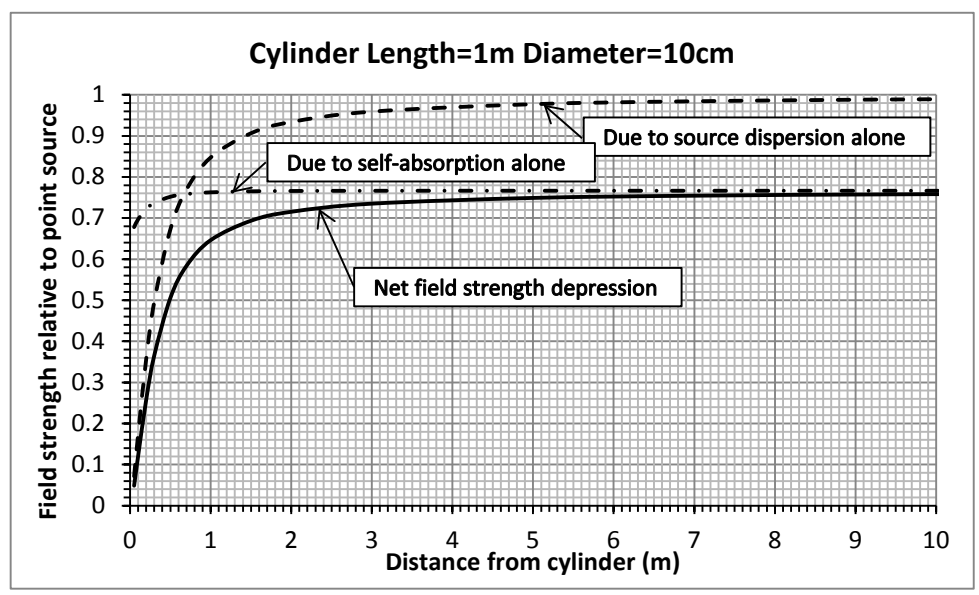

Figure 5. The field-strength depression due to dispersing emitters from a point source into a $10-\mathrm{cm}$ diameter cylinder 1 meter long. Emissions are assumed consistent with the inverse-square law. Absorption is computed using $\mathrm{HVL}=10 \mathrm{~cm}$. 


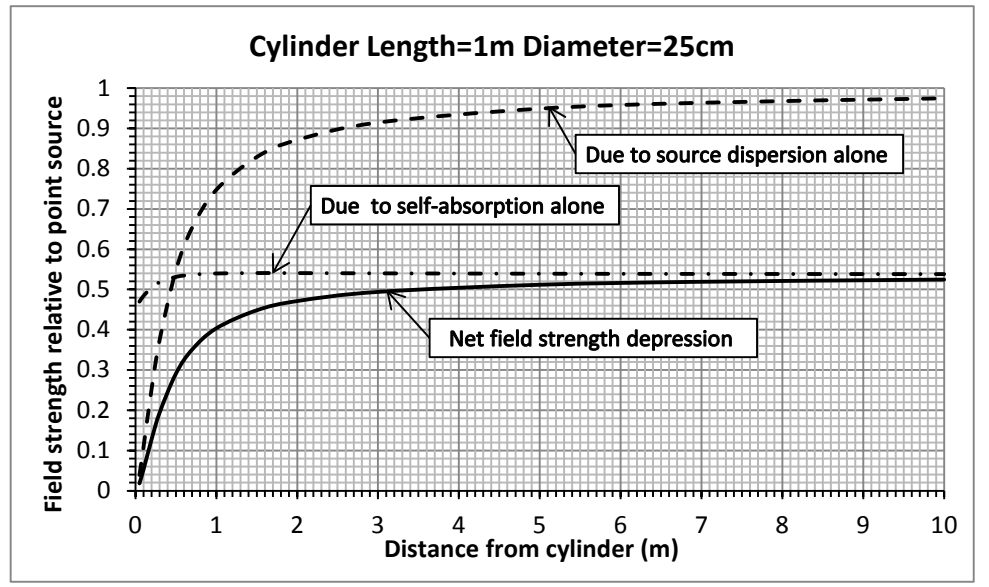

Figure 6. The field-strength depression due to dispersing emitters from a point source into a $25-\mathrm{cm}$ diameter cylinder 1 meter long. Emissions are assumed consistent with the inverse-square law. Absorption is computed using $\mathrm{HVL}=10 \mathrm{~cm}$.

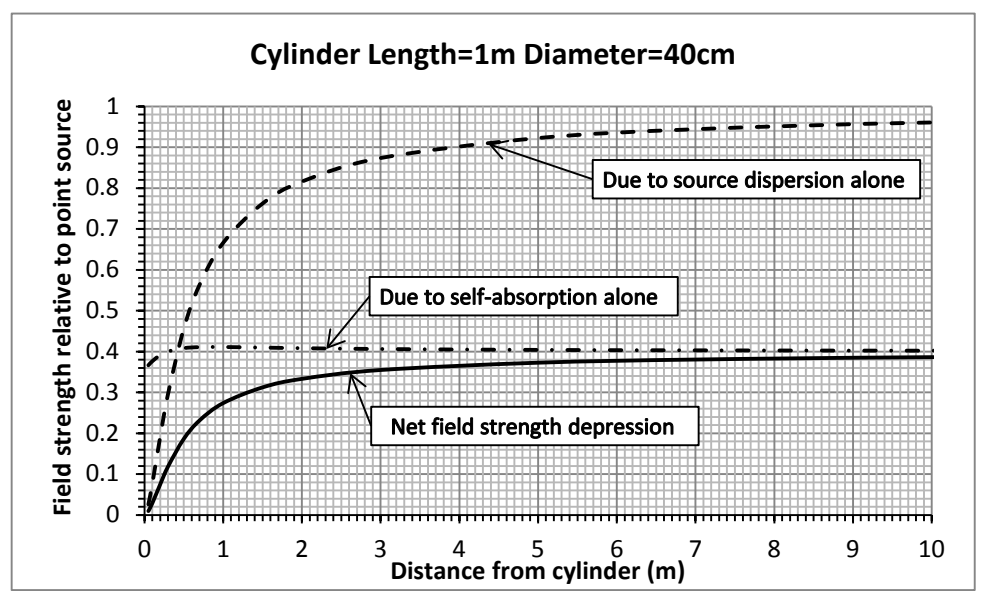

Figure 7. The field-strength depression due to dispersing emitters from a point source into a $40-\mathrm{cm}$ diameter cylinder 1 meter long. Emissions are assumed consistent with the inverse-square law. Absorption is computed using $\mathrm{HVL}=10 \mathrm{~cm}$.

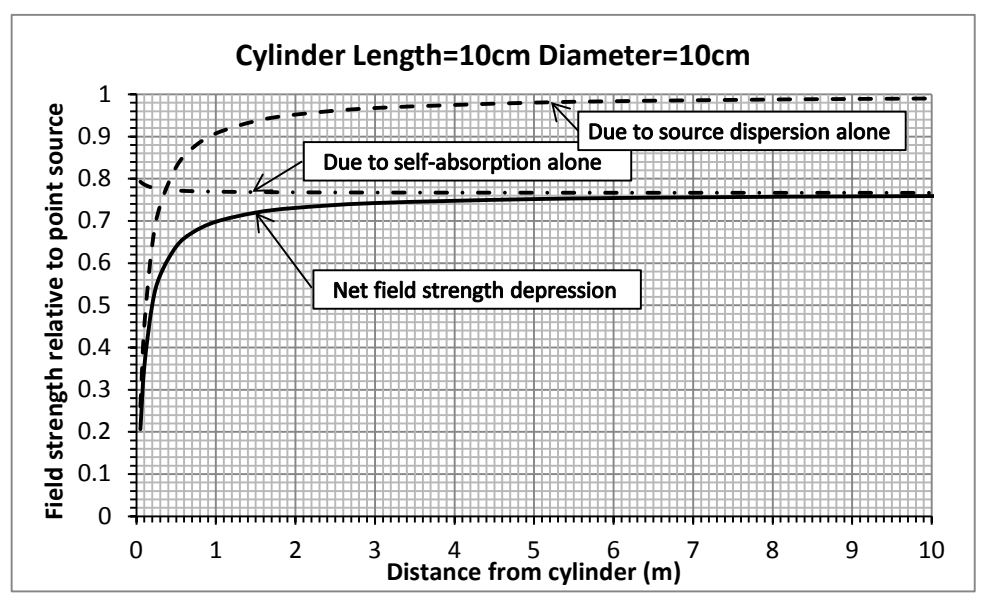

Figure 8. The field-strength depression due to dispersing emitters from a point source into a $10-\mathrm{cm}$ diameter cylinder $10 \mathrm{~cm}$ long. Emissions are assumed consistent with the inverse-square law. Absorption is computed using $\mathrm{HVL}=10 \mathrm{~cm}$. 


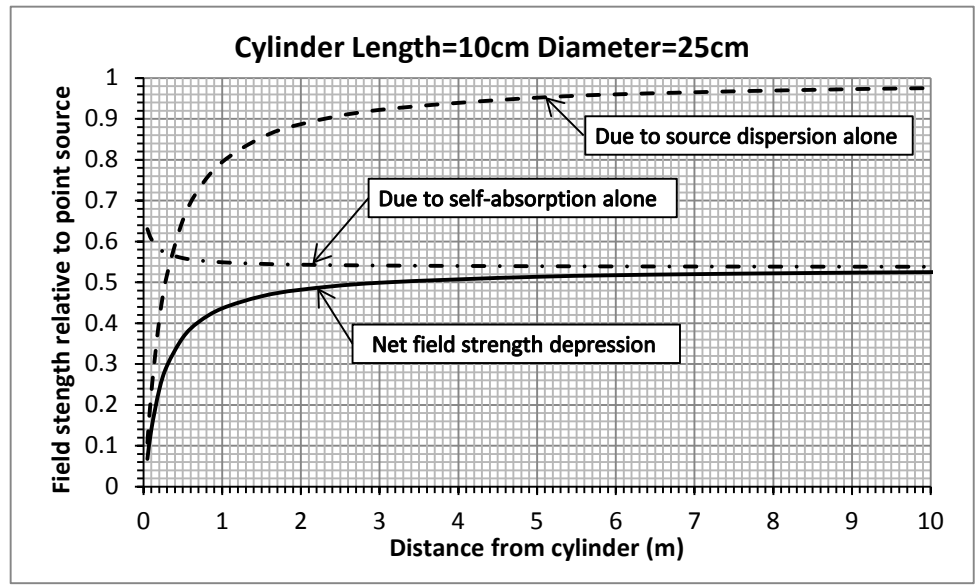

Figure 9. The field-strength depression due to dispersing emitters from a point source into a $25-\mathrm{cm}$ diameter cylinder $10 \mathrm{~cm}$ long. Emissions are assumed consistent with the inverse-square law. Absorption is computed using $\mathrm{HVL}=10 \mathrm{~cm}$.

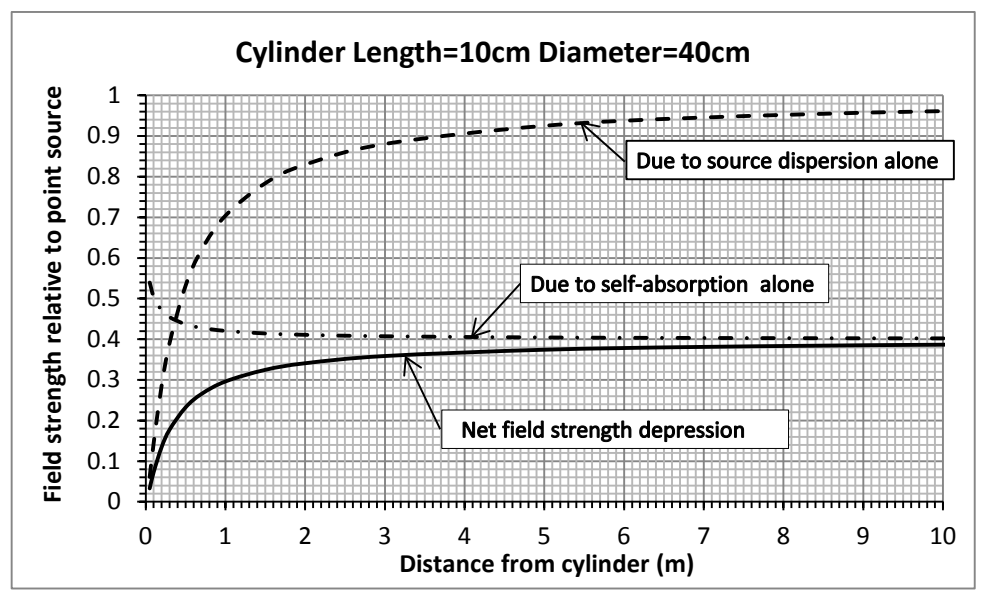

Figure 10. The field-strength depression due to dispersing emitters from a point source into a $40-\mathrm{cm}$ diameter cylinder $10 \mathrm{~cm}$ long. Emissions are assumed consistent with the inverse-square law. Absorption is computed using $\mathrm{HVL}=10 \mathrm{~cm}$.

The dispersion component of field-strength depression is valid for all emitters for which emissions are isotropic (provides inverse-square behavior). Figures 2-10 show that for all cylinders this is the dominant component of field-strength depression for small distances (less than about $50 \mathrm{~cm}$ ). This is because the inverse-square law dramatically amplifies the effect of dispersing the source when the field strength is assessed in close proximity to the cylinder. As the distance to field-strength assessment increases this dispersion component of the depression asymptotically approaches 1.0, indicating that, mathematically, the extended volume source is looking more and more like a point.

The self-absorption component of field-strength depression behaves differently. When the field strength is assessed in close proximity to the cylinder (Figure 11, assessment point B), two phenomena compete. The first phenomenon is a pronounced increase the field-strength depression as the assessment point approaches the cylinder wall (Figure 12 curve labelled "Length $=1 \mathrm{~m}$ "). It 


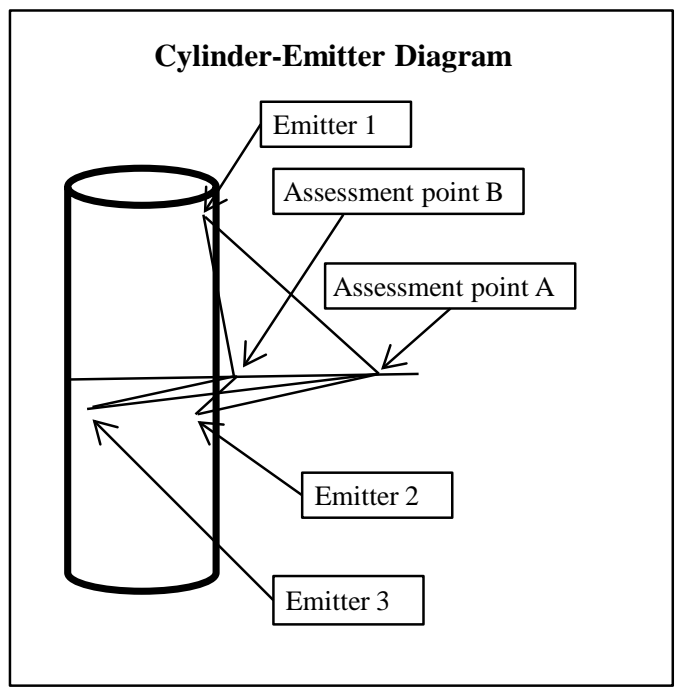

Figure 11. Depicts a cylinder with locations of some emitters and assessment points identified to assist explanations in the text.

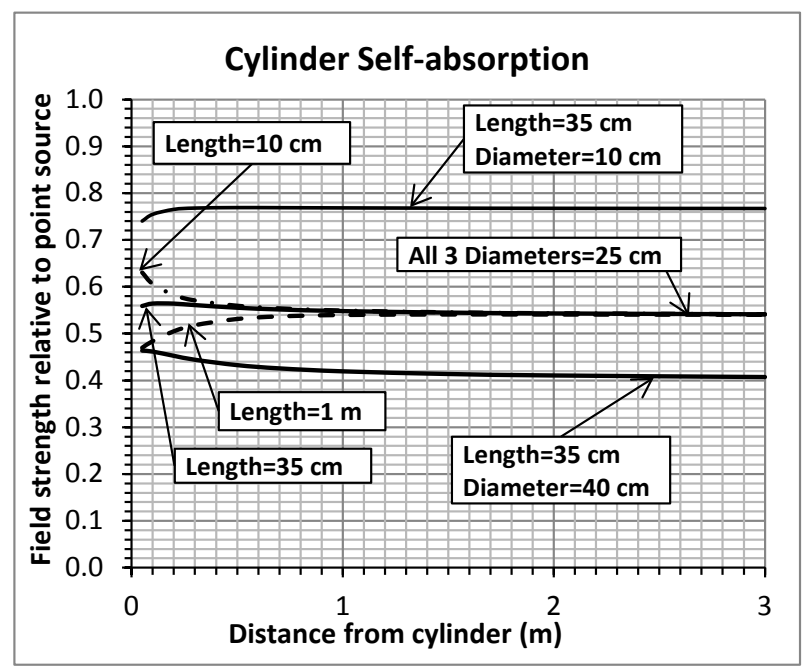

Figure 12. Demonstrating the 2 competing phenomena regarding the self-absorption component of field-strength depression as discussed in the text.

is most readily observed for long cylinders and is caused by emissions originating at the ends of the cylinder being required to travel exceedingly long distances within the absorbing media of the cylinder in order to reach the assessment point (Figure 11 assessment point $\mathrm{B}$ ). The second phenomenon is a pronounced decrease in the field-strength depression as the assessment point approaches the cylinder wall (Figure 12 curve labelled "Length $=10 \mathrm{~cm}$ "). It is most readily observed in short cylinders and is caused by the inverse square law's dramatically amplifying the influence of proximal emissions over that of distal emissions. As the assessment point approaches the cylinder wall, this effect results in distal emissions contributing hardly at all to the field strength compared to that of proximal emissions. This leads to what may appear to be a counterintuitive observation that: although the self-absorption of the distal emissions is much 
greater than that of the proximal emissions, the overall influence of the relatively small self-absorption of proximal emissions upon the field-strength assessed at the assessment point far exceeds that of the much greater self-absorption of the distal emission.

The second phenomenon decreases the field-strength depression assayed close to the cylinder wall, while the first phenomenon increases in the field-strength depression. Both of these phenomena are illustrated in Figure 12. Notice that for a $25-\mathrm{cm}$ diameter cylinder that is $35 \mathrm{~cm}$ long, the two phenomena cancel one another, keeping the field strength nearly constant as the assay approaches to within $5 \mathrm{~cm}$ of the cylinder wall. By shortening the cylinder to $10 \mathrm{~cm}$, emitters near the ends of the cylinder, whose emissions would have traveled considerable distances within the cylinder to reach the field-strength assessment point, are removed (Figure 11, Emitter 1 is gone), so that the second phenomenon dominates and the depression decreases as the cylinder wall is approached. By lengthening the cylinder to $1 \mathrm{~m}$ the number of emissions near the ends of the cylinder is increased so the first phenomenon dominates and the depression increases as the cylinder wall is approached. Similarly, Figure 12 (Length $=35 \mathrm{~cm}$, Diameter $=10 \mathrm{~cm}$ vs. Length $=35 \mathrm{~cm}$, Diameter $=40 \mathrm{~cm})$ shows that when holding the cylinder length constant at $35 \mathrm{~cm}$, by increasing the cylinder diameter to $40 \mathrm{~cm}$, thus increasing the inverse square's proximal to distal amplification differential, the second phenomenon dominates, and by decreasing the diameter to $10 \mathrm{~cm}$ decreases the inverse square effect, so the first phenomenon dominates.

As the distance to the assessment point increases, the self-absorption curve appears to plateau. However, technically it never plateaus because the second phenomenon is still operable at great distances. That is to say, when the cylinder to assessment point distance is sufficiently large compared to the cylinder dimensions, the inverse square's proximal to distal emitter amplification differential will appear to be imperceptible, but the increase in field-strength depression with increasing distance can be observed as long as Equation (7) is evaluated to a sufficient number of significant numbers, i.e., as long as the cylinder can mathematically be differentiated from a point.

\section{Conclusions}

Four general trends displayed in Figures 2-10 are, first: within $50 \mathrm{~cm}$ of the cylinder, field-strength depression is dominated by the dispersion component; second: as this distance increases the dispersion component gradually disappears (asymptotically approaches 1.0); third: the total depression then asymptotically approaches the self-absorption component; fourth: as the assessment point leaves the cylinder wall, the self-absorption component of the field-strength depression either initially decreases (first phenomenon above) or increases (second phenomenon) then appears to plateau, but, as discussed above, technically continues to increase indefinitely.

The data displayed in Figures 2-10 can be put to practical use. For example, if 
emitters were dispersed into a cylindrical volume $25 \mathrm{~cm}$ in diameter and $1 \mathrm{~m}$ long (approximating a small human or other animals), from Figure 6 the field-strength depression $20 \mathrm{~cm}$ from the cylinder would be about 0.25 from dispersion alone (Figure 6, "Due to source dispersion alone"), while, if the emitters were ${ }^{131} \mathrm{I}$, the total depression would be about 0.13 (Figure 6, "Net field strength depression"). Thus the field strength $20 \mathrm{~cm}$ from the cylinder for any emitter must be less than $25 \%$ of a simple point-source computation (Equation (3)), and, if the emitters were ${ }^{131} \mathrm{I}$, the measureable field strength would be approximately $13 \%$ of the point-source computation.

\section{Acknowledgements}

I am indebted to my wife, Karen L. Beetham, Ph.D., for rereading the manuscript multiple times and making many suggestions to substantially improve clarity and readability.

\section{Conflicts of Interest}

The author declares no conflicts of interest regarding the publication of this paper.

\section{References}

[1] Steward, P.G. (2017) An Excel-Based System to Manage Radiation Safety for the Family of Patients Undergoing ${ }^{131} \mathrm{I}$ Therapy. Journal of Nuclear Medicine Technology, 45, 102-110. https://doi.org/10.2967/jnmt.116.185322

[2] Steward, P.G. (2019) Radiation Field Strengths near Cylindrical Volume Sources via Point-Source Correction Factors. Health Physics Journal, 116, 60-68. https://doi.org/10.1097/HP.0000000000000952

[3] Dewji, S.A., Bellamy, M., Hertel, N., Leggett, R., Sherbini, S., Saba, M. and Eckerman, K. (2015) Assessment of the Point-Source Method for Estimating Dose to Members of the Public from Exposure to Patients with ${ }^{131} \mathrm{I}$ Thyroid Treatment. Health Physics Journal, 109, 233-241. https://doi.org/10.1097/HP.0000000000000327

[4] Wachsman and Drexler (2012) Average Half-Value and Tenth-Value Layers of Shielding Materials (Broad Beams). In: Johnson, T.E., Birky, B.K., Eds., Health Physics and Radiological Health, 4th Edition, Wolters Kluwer | Lippincott Williams \& Wilkins, 305. 